\title{
Relato do processo de resgate tipográfico: La Geografia, do século 16
}

\author{
Reporting a type revival: 16th Century's La Geografia
}

Laura Linck, Eduardo A B M Souza, Josinaldo Barbosa da Silva

tipografia, design de tipos, resgate tipográfico

Realizamos o resgate tipográfico do conjunto de caracteres itálicos em caixa-baixa de um exemplar do livro La Geografia, de 1564, do acervo da Biblioteca do Instituto Ricardo Brennand. Inicialmente, revisamos a bibliografia acerca do processo de resgate tipográfico e da história da tipografia. Assim, identificamos o tipógrafo Pierre Haultin como referencial. Para o desenho, utilizamos imagens das páginas do livro e referências de outras fontes digitais. Com auxílio da derivação, criamos alguns caracteres inexistentes no livro. Então, seguimos com a definição dos espaçamentos entre letras utilizando o método de Walter Tracy. Como resultado, obtivemos a fonte itálica caixa-baixa La geografia com 150 caracteres, assim como o levantamento bibliográfico e a descrição de etapas para a realização deste resgate tipográfico.

typography, type design, type revival

We carried out a typographic revival project of the lowercase set of italic characters of a copy of the book La Geografia, from 1564, from the collection of Ricardo Brennand Institute's library. Initially, we made a literature review of type revival processes and history of typography. Thus, we identified typographer Pierre Haultin's work as a reference. To draw characters, we used images of the book and references from other digital sources. With the aid of the derivation, we created characters absent in the book. So, we proceeded to spacing letters using Walter Tracy's method. As a result, we obtained the Italic lowercase font La geografia with 150 characters, as well as a literature review and the description of steps for the realization of this typographic revival.

\section{Introdução}

A configuração visual da linguagem verbal é influenciada pelos fatores históricos em que está inserida. Embora as tecnologias sejam determinantes, as convenções e valores culturais também têm seu papel na perenidade dos desenhos de letras; por isso, o design vernacular se vale desses elementos para se referir "à expressão artística e técnica que genericamente caracteriza um lugar ou período histórico" (Meggs, 2009, p.617).

A interação entre as mudanças tecnológicas e a caracterização cultural dos desenhos de tipos sempre orientou aqueles que trabalhavam com letras a retomar tipos precedentes para dar continuidade a essa atividade. Por isso, a transposição digital desses desenhos é extremamente relevante. Um dos principais cursos de design de tipos na Academia Real de Curitiba | Brazil | 2021 
Artes Holandesa (KABK) fundamenta parte da sua formação nessa abordagem, para que os designers contemporâneos aprendam "importantes fundamentos do design de tipos e o contato com conhecimentos essenciais no campo da tipografia" (Lebedenco \& Campos, 2018).

\section{Referencial teórico}

O resgate tipográfico é um processo constante de imitação e inovação na história da tipografia, já que pode ser concebido como "um encontro de formas tradicionais com tecnologias atuais" (Celso, 2000, p.1). Os designers divergem acerca dos projetos desta natureza, chegando a questionar a relevância de realizar mais resgates (c.f. Lebedenco \& Neder, 2016). Todavia, concordamos com Balius (2017), que defende a produção de novas tipografias porque são "a voz da mensagem" e um "objeto cultural a serviço da sociedade", dada a relação de pertencimento à cultura do povo que a utilizará. $\mathrm{O}$ autor faz um paralelo entre a linguagem escrita e as relações humanas, estabelecendo uma ligação essencial entre elas.

Essa atividade é complexa pois, conforme Shaw \& Hoefler (2017), a essência do design de uma fonte é indescritível e capturá-la é uma realização muito subjetiva. Lebedenco \& Neder (2016) apontam que esses resgates podem buscar reproduzir suas referências fielmente, sendo uma "adaptação tecnológica de um design pertencente a um período histórico distante, criado em uma tecnologia ultrapassada" (p.65). Essa reprodução deve se aproximar tanto quanto possível do desenho original. Ainda assim, se estabelece uma relação de coautoria entre o tipo original e o resultado do resgate, já que mesmo a mera adaptação tecnológica implica escolhas projetuais.

Nesse sentido, apresentaremos o relato do desenvolvimento de uma fonte digital a partir do livro La Geografia de 1564, de Cláudio Ptolomeu Alexandrino, do acervo do Instituto Ricardo Brennand (IRB), em Recife-PE. Nosso principal objetivo é relatar a realização de uma prática de resgate (revival) tipográfico da fonte itálica caixa-baixa com 150 caracteres, apresentando o levantamento bibliográfico e as etapas pelas quais passamos.

\section{Metodologia}

A pesquisa seguiu as etapas mostradas na Figura 1. Especificamente para o desenho dos tipos, seguimos os apontamentos de Lebedenco \& Neder (2016), complementada por Paniagua (2015), além de estudos anteriores realizados por Lima, Silva, Coelho (2013) e Marques, Silva, Pena (2013) sobre o mesmo objeto. 
Figura 1: Esquema metodológico. Elaborado pelos autores

\begin{tabular}{|c|c|c|}
\hline Revisão de literatura & $\begin{array}{l}\text { Organização do } \\
\text { banco de dados }\end{array}$ & $\begin{array}{l}\text { Pesquisa em acervos } \\
\text { e bibliotecas }\end{array}$ \\
\hline \multirow[t]{2}{*}{ Leitura de textos e artigos } & $\begin{array}{l}\text { Seleção dos melhores tipos, } \\
\text { com base nas fotos já } \\
\text { coletadas previamente por } \\
\text { outro grupo de pesquisa }\end{array}$ & $\begin{array}{l}\text { Visita ao Instituto Ricardo Brennand } \\
\text { para coleta de melhores imagens } \\
\text { Seleção de páginas com } \\
\text { qualidade superior }\end{array}$ \\
\hline & Análise macro & \\
\hline $\begin{array}{l}\text { Seleção para } \\
\text { referência da fonte }\end{array}$ & $\begin{array}{l}\text { Desenvolvimento da } \\
\text { fonte }\end{array}$ & $\begin{array}{l}\text { Espacejamento e } \\
\text { finalização da fonte }\end{array}$ \\
\hline $\begin{array}{l}\text { Pierre Hautin e a fonte digital } \\
\text { Verdigris foram indicadas como } \\
\text { melhores referências visuais }\end{array}$ & $\begin{array}{l}\text { Impressão de imagens em papel A4 a } \\
\text { partir das melhores ocorências } \\
\text { selecinadas através da análise macro }\end{array}$ & $\begin{array}{l}\text { Método de Walter Tracy aplicado } \\
\text { pelo FontForge }\end{array}$ \\
\hline \multirow[t]{2}{*}{$\begin{array}{l}\text { Nova análise macro realizada } \\
\text { paralela à vetorização }\end{array}$} & $\begin{array}{l}\text { Modulação dos principais caracteres } \\
\text { para ralizar a derivação de suas formas }\end{array}$ & $\begin{array}{c}\text { Finalização através da observação } \\
\text { das manchas gráficas e da tabela } \\
\text { de kerning indicado por Cheng } \\
\text { (2006) e Adobe (1997) }\end{array}$ \\
\hline & $\begin{array}{l}\text { Utilização da Verdigris e da modulação } \\
\text { como referências para os caracteres } \\
\text { não presentes no livro }\end{array}$ & \\
\hline
\end{tabular}

Na seção a seguir, descreveremos cada uma dessas etapas, relatando as circunstâncias que levaram às tomadas de decisão.

\section{Descrição do projeto}

Após análises das imagens anteriormente coletadas por Lima, Silva, Coelho (2013) e Marques, Silva, Pena (2013), selecionamos e organizamos os caracteres tipográficos para o desenho da fonte. Para tal, utilizamos a metodologia de análise macro (Figura 3), proposta por Aragão (2016). A partir desse tratamento inicial, partimos para a seleção de referência para o desenho. Foram escolhidas ocorrências que apresentavam melhores definições nos contornos dos desenhos, observando terminais, bojo e definição dos traços, resultando em referências para os caracteres. Elas foram impressas em tamanho A4 e desenhadas à mão para visualização e estudos de sua anatomia. 
Figura 2: Detalhe da página do livro La Geografia do exemplar do Instituto Ricardo Brennand

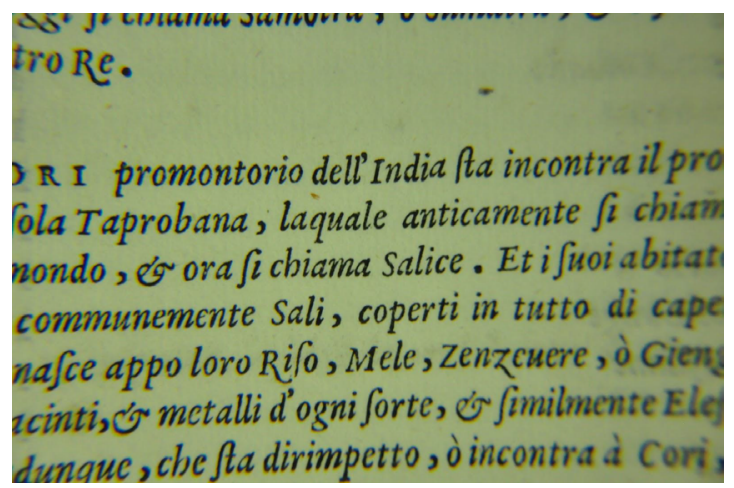

Figura 3. Exemplo da análise macro, elaborado pelos autores a partir da metodologia de Aragão (2016)

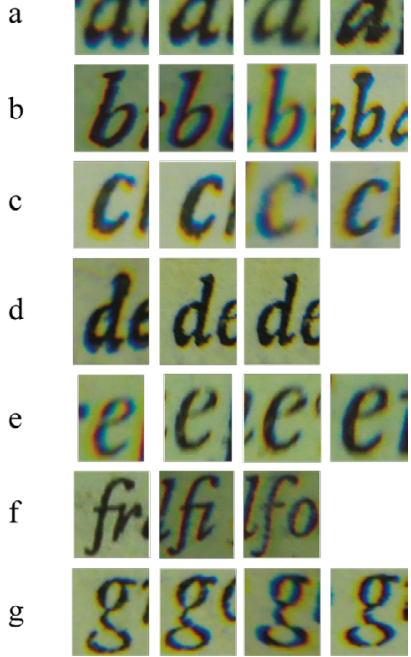

Entretanto, o material coletado anteriormente era de baixa resolução, logo inadequado para o redesenho dos caracteres. Assim, foi necessário realizarmos uma nova coleta de imagens em alta resolução de páginas do livro. Essas imagens foram cedidas pela Biblioteca do IRB, a partir de seleção prévia. Com as fotos em alta resolução, realizamos a análise macro novamente e partimos para a etapa de desenho digital em software vetorial.

Para operacionalizar o resgate, Shaw \& Hoefler (2017) auxiliam a compreender as decisões tomadas ao longo deste processo, comparando impressos originais e seus respectivos resgates digitais. Lebedenco e Neder (2016) dizem que é preciso analisar e comparar referências tipográficas de um mesmo período, para melhor compreender suas adaptações. Nessa busca por referências tipográficas renascentistas, constatamos que as tipografias de Pierre Haultin eram similares à tipografia utilizada no La Geografia. Além disso, as datas também eram compatíveis: o livro havia sido impresso em 1564 e Haultin produziu seus tipos sobretudo entre 1549 e 1581 (Vervliet, 2008). Dentre as variações observadas por Vervliet (ibid.), a Haultin's First Long Primer Italic se mostrou mais compatível à impressão do La Geografia, passando a ser utilizada como referência no processo de reconstrução. Tomamos também a fonte Verdigris como referência, pois é inspirada em trabalhos do século 16, como os 
de Robert Granjon, Hendrik van den Keere, e Pierre Haultin (Figuras 4 e 5). Isso auxiliou também na criação dos caracteres ausentes no La geografia e no desenho de alguns terminais.

Figura 4 - Verdigris de Mark van Bronkhorst, retirado do site da Adobe

\section{The quick brown fox jumps over a lazy dog.}

Figura 5 - Recorte do livro La Geografia de Claudio Ptolomeu, do Instituto Ricardo Brennand

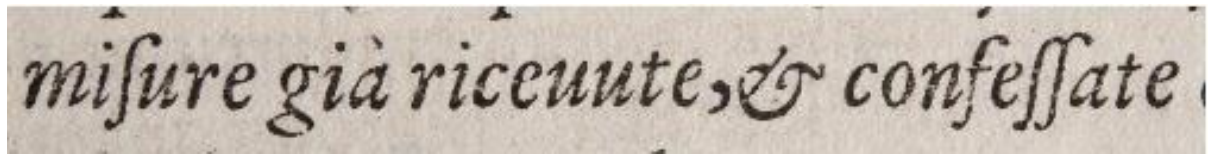

Observamos muita variedade nos caracteres impressos, que podem indicar falhas de impressão, tipos quebrados ou distribuição irregular de tinta. Essas variações são comuns na impressão com tipos mecânicos, conforme Aragão (2016). Essas anomalias foram minimizadas pela comparação com desenhos de outras tipografias similares, conforme revisão bibliográfica. Tais fatores foram considerados para a definição dos módulos dos caracteres para derivação.

Figura 6: Derivação de alguns caracteres. Elaborado pelos autores.

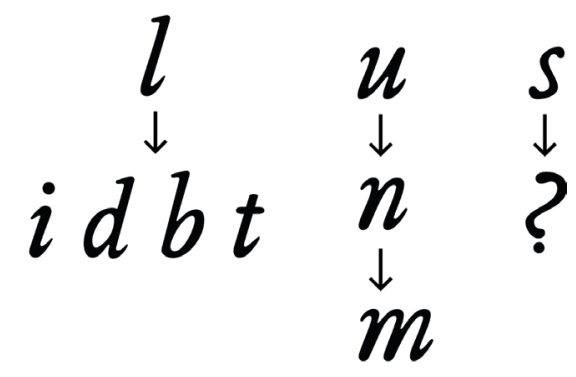

Inicialmente, os caracteres-chave $I$. $u$, s e $h$ foram desenhados a partir das imagens capturadas (Figura 6). Durante as análises, percebemos discrepâncias entre as ocorrências do mesmo caractere e no sistema de modulação dos tipos impressos. Por exemplo: a letra I apresenta vários ângulos de inclinação, ao passo que a inclinação as hastes de letras como $i$, $b, h$ e I são diferentes (Figura 7). Então, decidimos não aplicar o método de derivação, a fim de manter a textura do texto corrido proporcionado pela variação dos tipos de metal. 
Figura 7: Comparação da inclinação de caracteres do livro La Geografia. Elaborado pelos autores

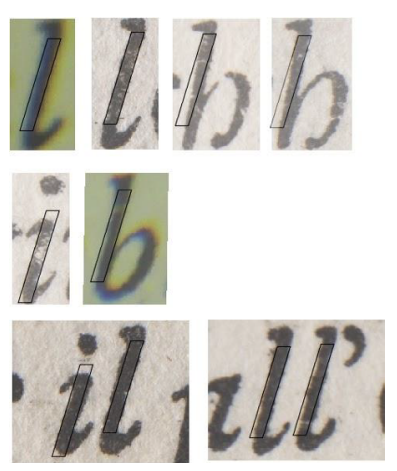

Ressaltamos que os caracteres faltantes, $j, w$ e alguns sinais diacríticos, foram desenvolvidos a partir de módulos dos caracteres, das imagens do livro (Figuras 8 e 9), além de referências da fonte Verdigris. Os desenhos foram realizados no software Adobe Illustrator e finalizados no FontForge, com ajustes nas formas (Figura 10), espacejamento, ligaturas e geração do arquivo fonte em opentype.

Figura 8: Comparação entre o caractere ij e a vetorização do j. Elaborado pelos autores<smiles>[AlH]</smiles>

Figura 9: Caracteres criados para melhor uso da fonte.Elaborado pelos autores

\begin{tabular}{|l|c|c|c|c|c|c|c|c|c|c|c|c|}
\hline$/$ & & $\backslash$ & $\ldots$ & - & $x$ & $\underline{a}$ & $\underline{1}$ & $\boldsymbol{j}$ & $\dot{2}$ & $\%$ & $\#$ \\
\hline$*$ & - & $\div$ & \pm & $=$ & + & $\langle>$ & $\ll \gg$ & $\wedge$ & emdash & endash & {[]} & () \\
\hline
\end{tabular}

Figura 10: Vetorização no Illustrator e ajustes no FontForge. Elaborado pelos autores

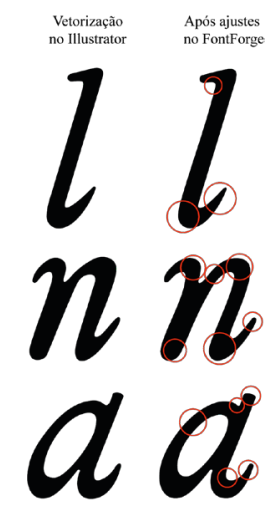


Para a definição do espacejamento, utilizamos o método proposto por Walter Tracy (2003) (Figura 11), complementado pela tabela de Cheng (2006) para os kernings. O processo foi orientado pelos testes de impressão conforme modelo da Adobe (1997) e de páginas de textos. Estes testes permitiram verificar a leiturabilidade da fonte, funcionamento de descendentes e ascendentes, textura da página, entre outros fatores. Como resultados dessa etapa tivemos a troca do caractere $\boldsymbol{s}$ longo para o $\boldsymbol{s}$ simples, a adição de ligaturas e mudanças na angulação de descendentes. (Figuras 12 e 13)

Figura 11: Método retirado de espacejamento de Walter Tracy, 2003
${ }_{1}^{1} \mathrm{~b}_{5} \quad{ }_{5} \mathrm{c}_{6} \quad{ }_{5} \mathrm{~d}^{1} \quad{ }_{5} \mathrm{e}_{6} \quad{ }_{3} \mathrm{~h}^{2}$
31 Igual ao lado esquerdo do $n$
3 Um pouco mais do que o lado esquerdo do $n$
$\mathbf{j}_{1}{ }^{1}{ }^{3} \mathrm{~K}_{4} \quad{ }^{3} \mathbf{1}^{1} \quad{ }^{1} \mathrm{~m}^{2} \quad{ }_{3} \mathrm{p}^{5}$
4 Espaço mínimo
${ }^{4} \mathrm{~V}^{4}$
${ }^{4} \mathrm{~W}^{4} \quad{ }^{4} \mathrm{Y}^{4}$

a $\mathrm{f} \mathrm{g} \mathrm{s} \mathrm{t} \mathrm{Z}$ devem ser espaçados opticamente, conforme padrões

Figura 12: Comparação entre $s$ longo e $f$. Elaborado pelos autores

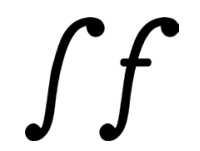

Figura 13: Ligaturas. Elaborado pelos autores

\section{fifl fr ft fp fifh fifflffh ffi st sp ct}

\section{Discussão}

Obtivemos a fonte digital La Geografia em caixa-baixa itálica, com 150 caracteres, que preserva o desenho e a textura irregular da mancha do livro de 1564. Conhecer o contexto e os tipos de metal da época - além de outras fontes digitais que também resgatam essas características - auxiliou na definição do resgate dos caracteres e no desenvolvimento dos que faltavam. Quanto ao processo, salientamos que, pela sua natureza, alguns problemas só surgem ao longo do desenvolvimento do projeto. Por exemplo, a definição dos ângulos de inclinação do desenho de alguns caracteres.

Portanto, acreditamos que este projeto contribui para a prática de resgate tipográfico por meio do levantamento bibliográfico direcionado para a realização desses projetos, acompanhado do relato das etapas executadas. Sobretudo, reiteramos a importância da pesquisa histórica e da prática do resgate para o ensino de tipografia. Com o relato aqui exposto, esperamos auxiliar futuros trabalhos na área. 


\section{Referências}

ADOBE Systems Incorporated. (1997). Designing multiple master typefaces. https://adobe-typetools.github.io/font-tech-notes/pdfs/5091.Design_MM_Fonts.pdf

Aragão, I. (2016). Tipos móveis de metal da Funtimod: Contribuições para a história tipográfica brasileira [Tese de doutorado]. Universidade de São Paulo.

Balius, A. (2017). El valor de la tipografia. In A. Balius (Ed.), Anuario Lletraferits 2017: Reflexiones en torno a la letra.

Lo Celso, A. (2000). A discussion on Type Design Revivalism [Dissertação de mestrado]. University of Reading.

Lebedenco, É., \& Neder, R. (2016). Fundamentos do resgate tipográfico. DAT Journal, 1(1), 52-70.

Lebedenco, É. C., \& de Campos, G. B. (2018). O resgate tipográfico na educação do designer de tipos no curso de mestrado type and media (KABK). 22, 14.

Lima, C. L. F. de, Barbosa, J., \& Coelho, E. (2013). Criação de fontes digitais a partir do estudo tipo gráfico do livro do século XVI, La Geografia, de Claudio Ptolomeo Alessandro. In: VIII Congresso de Iniciação Científica do Instituto Federal de Pernambuco, Recife.

Marques, C. L., Silva, J. B. da, \& Pena, S. da S. (2013). O livro do século XVI: apreciação dos recursos tipográficos utilizados na produção do livro La Geografia impresso em 1564. In Anais do VIII Congresso de Iniciação Científica do IFPE. Didática Editora do Brasil.

Meggs, P. B., \& Purvis, A. W. (2009). História do design gráfico. Cosac Naify.

Paniagua, J. M. R. (2015). Ibarra Real. Un modelo metodológico para el redibujo y reconstrucción de tipografías clásicas. Del plomo al vector [Tesis doctoral]. Universidad Complutense de Madrid.

Shaw, P., \& Hoefler, J. (2017). Revival type: Digital typefaces inspired by the past. Yale University Press.

Tracy, W. (2003). Letters of credit: A view of type design. D.R. Godine.

Vervliet, H. D. L. (2008). The palaeotypography of the French Renaissance: Selected papers on sixteenth-century typefaces. Brill.

\section{Sobre os autores}

Laura Linck Maciel; IFPE; Recife, Recife, Pernambuco, Brasil; linck.lauram@gmail.com

Eduardo A B M Souza; Mestre; IFPE/UFPE; Recife, Pernambuco, Brasil;

eduardosouza@recife.ifpe.edu.br

Josinaldo Barbosa Da Silva; Doutor; IFPE/UFPE; Recife, Pernambuco, Brasil;

josinaldobarbosa@recife.ifpe.edu.br 